\title{
Properties of electrons near a Van Hove singularity
}

\author{
M. A. H. Vozmediano ${ }^{1}$, J. González ${ }^{2}$, F. Guinea ${ }^{3}$, J. V. Alvarez ${ }^{4}$, and B. Valenzuela 3 \\ 1 Departamento de Matemáticas, Universidad Carlos III, Avenida de la Universidad 30, Leganés. 28913 Madrid, Spain \\ ${ }^{2}$ Instituto de Estructura de la Materia, Consejo Superior de Investigaciones Científicas, Serrano 1223, 28006 Madrid, Spain \\ ${ }^{3}$ Instituto de Ciencia de Materiales, Consejo Superior de Investigaciones Científicas, Cantoblanco, 28049 Madrid, Spain \\ Universität des Saarlandes, D-66041 Saarbrücken, Germany
}

(October 27, 2018)

\begin{abstract}
The Fermi surface of most hole-doped cuprates is close to a Van Hove singularity at the M point. A two-dimensional electronic system, whose Fermi surface is close to a Van Hove singularity shows a variety of weak coupling instabilities. It is a convenient model to study the interplay between antiferromagnetism and anisotropic superconductivity. The renormalization group approach is reviewed with emphasis on the underlying physical processes. General properties of the phase diagram and possible deformations of the Fermi surface due to the Van Hove proximity are described.
\end{abstract}

71.18.+y, 71.10.Hf, 74.25.Ha.

The Fermi surface of most hole-doped cuprates is close to a Van Hove singularity at the M point. The possible relevance of this fact to the superconducting transition as well as to the anomalous behavior of the normal state was put forward in the early times of the cuprates and gave rise to the so-called Van Hove scenario (VHS) [1]. Early critiques to the scenario have been confronted with the observation by angle resolved photoemission (ARPES) of very flat bands near the Fermi level [2] and with the consistency of most data with the VHS. Van Hove singularities are found nowadays in a vaste number of experimental and theoretical papers under various names as hot spots or flat bands.

The field theory renormalization group techniques adapted to condensed matter problems in [3], have been crucial to elucidate issues such as the renormalization of the quasiparticle peak and of the Fermi surface shape as well as to provide a clear picture of the phase diagram in the proximity of large peaks in the density of states.

In this paper we give an updated review of the VHS and put forward some new features that can be explained within this framework.

A two dimensional electronic system with a square lattice has typically two inequivalent high symmetry points in the Brillouin Zone where a saddle point in the dispersion relation is likely to occur. Near a Van Hove singularity the fermion density of states diverges, so that even arbitrarily weak interactions can produce large effects. When the Fermi level reaches these points, a variety of response functions diverge.

The simplest microscopic model to study this problem is the $t-t^{\prime}$-Hubbard model which has the dispersion relation

$$
\begin{aligned}
\varepsilon(\mathbf{k}) & =-2 t\left[\cos \left(k_{x} a\right)+\cos \left(k_{y} a\right)\right] \\
& -2 t^{\prime} \cos \left(k_{x} a\right) \cos \left(k_{y} a\right)-\mu-2 t^{\prime},
\end{aligned}
$$

Band structure calculations 四 provide values for the parameters that can accurately describe the observed Fermi surface of most cuprates. For the hole-doped materials, $t^{\prime}<0$ and, in all cases, $t^{\prime}<t / 2$. Typical values for BISCO are $t \sim 0.5 \mathrm{eV}, t^{\prime} / t \sim-0.3$, and a small value of t" that we shall ignore here.

This dispersion relation has two inequivalent saddle points at $\mathrm{M}(\pi, 0)$ and $\mathrm{M}^{\prime}(0, \pi)$. The Van Hove model in its simpler formulation is obtained by assuming that for fillings such that the Fermi line lies close to the singularities, the majority of states participating in the interactions will come from regions in the vicinity of the saddle points. Taylor expanding eq. (II) around the two points gives the effective relation

$$
\varepsilon_{A, B}(\mathbf{k}) \approx \mp\left(t \mp 2 t^{\prime}\right) k_{x}^{2} a^{2} \pm\left(t \pm 2 t^{\prime}\right) k_{y}^{2} a^{2},
$$

which provides a well defined continuous field theory model to which renormalization group techniques can be rigorously applied [5].

Particle-particle and particle-hole susceptibilities have logarithmic divergences giving rise to various instabilities at low temperatures. The renormalization group allows to study the flow of the various response functions and to get a phase diagram as a function of t' and $U$.

In the simplest of local repulsive interactions, the leading instabilities are towards ferro- and antiferromagnetism, and also towards d-wave superconductivity [6]. The phase diagram is shown in fig. 1.

It is interesting to remark that the electron-hole susceptibility at $\overrightarrow{\mathbf{Q}}=(\pi, \pi)$ also diverges logarithmically, $\chi_{e h}(\overrightarrow{\mathbf{Q}}) \sim \log \left(\Lambda / \epsilon_{F}\right)$, where $\Lambda$ is a high energy cutoff, of the order of the bandwidth, $\Lambda \sim 1 \mathrm{eV}$, and $\epsilon_{F}$ is the distance of the Fermi level to the saddle point, which is typically one order of magnitude or more smaller. The ferromagnetic transition, generic of two dimensional models of this type [7], is due to scattering processes which involve small momentum transfer. These processes are, most likely, screened in a 3D structure, where scattering with $|\overrightarrow{\mathbf{q}}| \sim d^{-1}$, where $d$ is the interlayer distance, is strongly modified.

From the critical frequencies separating the antiferromagnetic and the superconducting region in fig. 1, we 
can see that the critical temperature for superconductivity increases with the value of t', a result in agreement with recent band theory calculations $[8]$.

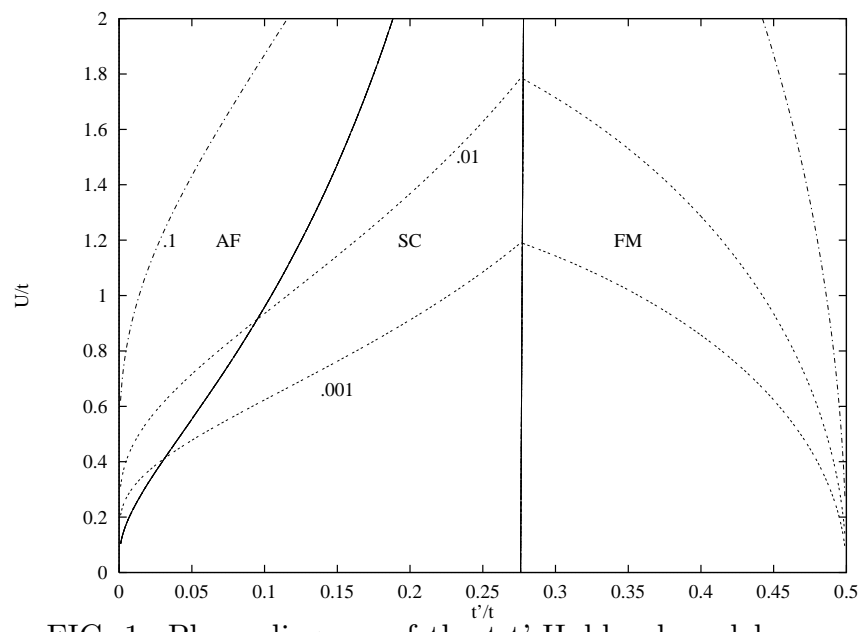

FIG. 1. Phase diagram of the t-t'-Hubbard model near a Van Hove singularity from ref. 6. The dotted lines are contour lines corresponding to the critical frequencies shown in the figure.

Within the same model we can obtain a number of other features consistent with the observed behavior of the cuprates: i) The normal state has anomalous features, including a quasiparticle lifetime which goes as $|\epsilon|$ [9,10], ii) For some values of the parameters, stripes are found [11,12], and iii) the out of plane electron hopping is incoherent 13].

The proximity of the Fermi surface to Van Hove singularities induce strong renormalization of the Fermi surface shape which can give rise to deformations of the Fermi surface breaking the lattice symmetry 16. An extended interaction is needed for this to occur 117 .

The competition between d-wave superconductivity and an instability at $\overrightarrow{\mathbf{Q}}$ leads to three possible scenarios:

i) The d-wave susceptibility, which diverges as $\chi_{e-e}(\omega) \sim \log (\Lambda / \omega) \log \left(\Lambda / \epsilon_{F}\right)$, diverges first, before instabilities at $\overrightarrow{\mathbf{Q}}$ develop. There is a transition to a dwave superconductor. This is probably realized in the overdoped regime.

ii) The SDW instability at $\overrightarrow{\mathbf{Q}}$ diverges much faster than the superconducting one. The ground state is antiferromagnetic. This is likely to occur near half filling.

iii) Both instabilities are comparable. The ground state will be, most likely, a d-wave superconductor. The reason is that, in the absence of perfect nesting, the instability at $\overrightarrow{\mathbf{Q}}$, even if fully developed, does not lead to an insulating state. Portions of the Fermi surface remain gapless. The logarithmic divergences in the BCS channel are not fully suppressed, and become $\chi_{e-e}(\omega) \sim$ $\log (\Lambda / \omega) \log \left(\Lambda / \Delta_{\overrightarrow{\mathbf{Q}}}\right)$, where $\Delta_{\overrightarrow{\mathbf{Q}}}$ is a scale determined by the divergence of $\chi_{e-h}(\overrightarrow{\mathbf{Q}})$. The existence of anomalous behavior at a scale above the superconducting critical temperature has been widely reported in the underdoped regime, and is usually associated to the pseudogap.

In the case where the Fermi surface does not have space symmetries, the phase diagram shows competition between ferromagnetism and p-wave superconductivity 14]. Coexistence of ferromagnetism and triplet superconductivity has been reported in $\mathrm{MgCNi}_{3}$ [18] whose Fermi surface lies close to Van Hove singularities [19], and in the ruthenium compounds [20].

As a summary we list the main results obtained by the combined RG techniques in the VHS which have some experimental support.

- vanishing of the quasiparticle peak,

- pinning of the Fermi surface at the singularity,

- in-plane resistivity linear in frequency,

- incoherence of the c axis transport,

- deformation of the Fermi surface breaking the tetragonal symmetry of the lattice (Pomeranchuk instability),

- coexistence of antiferromagnetism d-wave superconductivity, and existence of a ferromagnetic phase if the Fermi surface is four fold symmetric,

- increasing of $T_{c}$ with $t^{\prime}$ in the t-t' model,

- coexistence of ferromagnetism and p wave superconductivity for asymmetric Fermi surfaces.

[1] J. Labbé and J. Bok, Europhys. Lett. 3 (1987) 1225. J. Friedel, J. Phys. (Paris) 48 (1987) 1787; 49 (1988) 1435. H. J. Schulz, Europhys. Lett. 4, 609 (1987). R. S. Markiewicz and B. G. Giessen, Physica (Amsterdam) 160C (1989) 497. R. S. Markiewicz, J. Phys. Condens. Matter 2 (1990) 665. D. M. Newns et al., Phys. Rev. Lett. 69, 1264 (1992).

[2] D. S. Dessau et al., Phys. Rev. Lett. 71, 2781 (1993), D. M. King et al., Phys. Rev. Lett. 73, 3298 (1994), K. Gofron et al., Phys. Rev. Lett. 73, 3302 (1994). See Z.H. Shen et al., Science 267, 343 (1995), and references therein.

[3] R. Shankar, Rev. Mod. Phys. 61, 433 (1989).

[4] W. E. Pickett, Rev. Mod. Phys. 66, 129 (1994).

[5] J. González, F. Guinea and M. A. H. Vozmediano, Nucl. Phys. B485, 694 (1997).

[6] J. V. Alvarez, J. González, F. Guinea and M. A. H. Vozmediano, J. Phys. Soc. Jpn. 67, 1868 (1998).

[7] R. Hlubina, S. Sorella and F. Guinea, Phys. Rev. Lett. 78, 1343 (1997).

[8] E. Pavarini, I. Dagupta, T. Saha-Dagupta, O. Jepsen, and $\mathrm{O}$. K. Andersen, this proceedings.

[9] P. A. Lee and N. Read, Phys. Rev. Lett. 58, 2691 (1987).

[10] J. González, F. Guinea and M. A. H. Vozmediano, Europhys. Lett., 34, 711 (1996).

[11] B. Valenzuela, M. A. H. Vozmediano, and F. Guinea, Phys. Rev. B 62, 11312 (2000). 
[12] B. Normand and A. Kampf, preprint (cond-mat/0102201).

[13] M. A. H. Vozmediano, F. Guinea and M. P. LópezSancho, to be published.

[14] J. González, F. Guinea and M. A. H. Vozmediano, Phys. Rev. Lett. 84, 4930 (2000).

[15] A. Himeda and M. Ogata, preprint (cond-mat/0003278.

[16] C. J. Halboth and W. Metzner, Phys. Rev. Lett. 85, 5162 (2000).

[17] B. Valenzuela and M. A. H. Vozmediano, Phys. Rev. B. 63, 153103 (2001).

[18] T. He, et al., Nature 411, 54 (2001).

[19] H. Rosner, et al., preprint (cond-mat/0106583).

[20] R. Matzdorf, et al., Science 289, 746 (2000); K. M. Shen, et al., preprint (cond-mat/0105487). 